Studie zu nachhaltigkeitsorientierten Mitarbeitern

\title{
Nachhaltige Werte am Arbeitsplatz lohnen sich
}

\author{
Mitarbeiter wollen ihre persönlichen Überzeugungen auch am \\ Arbeitsplatz leben. Wenn sie damit Erfolg haben und wenn sie \\ ihren Arbeitgeber als sozial-ökologisch verantwortlich wahrnehmen, \\ werden zentrale Faktoren des Personalmanagements positiv \\ beeinflusst. Von Christoph Harrach, Ulf Schrader, Laura Stanszus \\ und Viola Muster
}

m Kontext des nachhaltigkeitsorientierten Personalmanagements bietet die Idee der Green Work-Life-Balance (Muster/Schrader 2011) eine neue Perspektive für die Integration des Nachhaltigkeitsgedankens in Organisationen. Dabei wird davon ausgegangen, dass es Wechselwirkungen zwischen einer privaten Nachhaltigkeitsorientierung von Mitarbeitern und der Arbeitswelt gibt.

Indem Unternehmen diese Wechselwirkungen bewusst fördern und die Mitarbeiter dazu befähigen, ihre sozial-ökologischen Ideen und Erfahrungen an den Arbeitsplatz zu bringen, können die Bindung, die Zufriedenheit und das Commitment dieser Mitarbeiter gesteigert und damit zentrale personalpolitische Ziele erreicht werden (Schrader/ Harrach 2013).

\section{Befragung von Arbeitnehmern}

Im Rahmen des von der Deutschen Bundesstiftung Umwelt geförderten Projektes „Nachhaltig leben und arbeiten“ wurden vom Fachgebiet Arbeitslehre/ Ökonomie und Nachhaltiger Konsum der Technischen Universität (TU) Berlin 3.042 Arbeitnehmer im deutschsprachigen Raum befragt. Die Befragten stammen aus drei Stichproben. Die erste Stichprobe umfasste 1.364 Teilnehmer der „Grünen Community“, rekrutiert in Selbstselektion über „grüne“ Leitmedien wie das Naturkost-Magazin Schrot \& Korn oder die Onlineplattform karma konsum.de. Die zweite Stichprobe be- stand aus einem online-repräsentativen Bevölkerungsdurchschnitt mit 1.079 Teilnehmern, rekrutiert über den Online-Panel-Anbieter respondi. Die dritte Stichprobe bestand aus Mitarbeitern der Praxispartner aus dem Lebensmitteleinzelhandel mit 599 Teilnehmern.

Die Studie zeigt auf, wie Ziele des Nachhaltigkeits- und Personalmanagements in Unternehmen durch die Einbindung privater Nachhaltigkeitsorientierung von Mitarbeitern besser erreicht werden können. Damit erweitert und konkretisiert die Befragung die Erkenntnisse sozialwissenschaftlicher Forschung zu Wechselwirkungen zwischen "Arbeit und Leben“ in Bezug auf sozialökologische Einstellungen und Verhaltensweisen.

\section{Werte im Arbeitsleben}

Die Untersuchung basiert auf der zentralen Hypothese, dass Menschen mit einer privaten Nachhaltigkeitsorientierung ihre Werte, Einstellungen und Verhaltensweisen auch in den beruflichen Alltag einbringen wollen. Die Ergebnisse bestätigen diese Annahme. Zur Operationalisierung der privaten Nachhaltigkeitsorientierung wurden die latenten Variablen „Umwelteinstellung“ nach Diekmann und Preisendörfer (2001) und eine an der TU Berlin erstellte Skala zum „Nachhaltigen Konsumverhalten“ genutzt.

Das Einbringen der privaten Werte in die Arbeitswelt wurde anhand der Dimension „Bedeutung“ des Konstrukts „Psychologisches Empowerment“ nach
Spreitzer (1995) operationalisiert, welche inhaltlich modifiziert wurde $\mathrm{zu}$ „Bedeutung, Nachhaltigkeitsorientierung am Arbeitsplatz einbringen zu können“. Die Daten zeigen eine sehr starke positive Korrelation zwischen der Umwelteinstellung und dieser Bedeutung $(\mathrm{r}=0,62)$ sowie einen stark positiven $\mathrm{Zu}$ sammenhang zwischen dem nachhaltigen Konsumverhalten und dieser Bedeutung $(r=0,39)$; beide Zusammenhänge sind hoch signifikant $(\mathrm{p}<0,001)$. Je mehr sich also ein Mitarbeiter im Privatleben nachhaltig orientiert, umso bedeutungsvoller ist es für ihn, diese Einstellungen und Verhaltensweisen auch am Arbeitsplatz einbringen zu können.

\section{Werte als Erfolgsfaktoren}

Die zweite Hypothese der Untersuchung war, dass das mehr oder minder erfolgreiche Einbringen der privaten Nachhaltigkeitsorientierung sich in personalpolitischen Erfolgsfaktoren widerspiegelt. Die Wirksamkeit beim Einbringen der Werte wurde durch die Dimension „Wirksamkeit“ des Konstruktes Empowerment gemessen, die auch hinsichtlich Nachhaltigkeit modifiziert wurde. Zur Operationalisierung der personalpolitischen Erfolgsfaktoren wurden die Konstrukte Arbeitszufriedenheit (nach Valentine/Fleischmann 2008), Commitment (nach Maier/Woschée 2002) und Mitarbeiterbindung (in Anlehnung an Forsyth/Polzer-Debruyne 2007) herangezogen.

Auch hier bestätigen die Daten die angenommenen Zusammenhänge mit positiver Korrelation ( $r=0,26$ für Zufriedenheit, $r=0,31$ für Bindung und $r=0,44$ für Commitment) auf einem hohen Signifikanzniveau $(\mathrm{p}<0,001)$. Das heißt, dass Mitarbeiter, die ihre private Nachhaltigkeitsorientierung am Arbeitsplatz erfolgreich einbringen können, zufriedener sind, eine höhere Bindung sowie ein höheres Commitment zu ihrem Arbeitgeber aufweisen.

Eine dritte Hypothese besagte, dass diese personalpolitischen Erfolgsfaktoren auch in einem positiven Zusammenhang mit den im Mitarbeiterkreis wahrge- 
nommenen Leistungen im Bereich Corporate Social Responsibility (CSR) eines Unternehmens stehen (operationalisiert in Anlehnung an Valentine/Fleischmann 2008). Auch dieser Zusammenhang kann mit den Befragungsdaten belegt werden. So weist die CSR-Wahrnehmung hochsignifikante, positive Korrelationen mit Zufriedenheit und Bindung auf $(\mathrm{r}=0,27)$; besonders stark ist die Korrelation mit dem Konstrukt Commitment ( $r=0,45)$. Je mehr ein Unternehmen sich also nach Einschätzung der eigenen Mitarbeiter für Umwelt und Gesellschaft einsetzt, umso höher ist die Zufriedenheit und Bindung dieser Mitarbeiter und umso größer ist ihre Bereitschaft, sich auch besonders für das Unternehmen einzusetzen. Dieses Ergebnis legt den Schluss nahe, dass CSR-Aktivitäten nicht nur gegenüber Kunden, sondern insbesondere auch gegenüber den eigenen Mitarbeitern kommuniziert werden sollten.

\section{Schlussfolgerungen für das Personalwesen}

Die Ergebnisse, die hier für die aggregierte Gesamtbefragung präsentiert wurden, sind in allen Teilstichproben ähnlich. Zusammenfassend unterstreichen sie die Wichtigkeit der Mitarbeiter für die nachhaltige Entwicklung von Unternehmen. Für die unternehmerische Praxis implizieren die Daten, dass sich eine gezielte Integration der Mitarbeiter in das Nachhaltigkeitsmanagement in wettbewerbsrelevanten Erfolgsfaktoren im Personalwesen widerspiegelt und sich somit ökonomisch auszahlen kann. Das Einbringen der privaten nachhaltigkeitsorientierten Werte, Einstellungen und Verhaltensweisen lässt sich relativ einfach über klassische Instrumente wie das betriebliche Vorschlagswesen oder Ideenwettbewerbe umsetzen. Auch der Aufbau von bereichsübergreifenden Arbeitsgruppen kann die nachhaltigkeitsorientierten Mitarbeiter in ihrer Wirksamkeit stärken.

Eine besondere Rolle für die Wirksamkeit der Mitarbeiter am Arbeitsplatz spielen die Vorgesetzten. Hier sollte weitere Forschung die Rahmenbedingun- gen für ein erfolgreiches Nachhaltigkeits-Empowerment untersuchen. Auch besteht im Bereich der Förderung von Nachhaltigkeitsinnovationen durch Beteiligung der eigenen Mitarbeiter weiterer Forschungsbedarf.

\section{Anmerkung}

Mehr Informationen zum Projekt im Internet unter www.nachhaltig-leben-und-arbeiten.de.

\section{Literatur}

Diekmann, A./Preisendörfer, P. (2001): Umweltsoziologie. Eine Einführung. Reinbek: Rowohlt.

Forsyth, S./Polzer-Debruyne, A. (2007): The organisational pay-offs for perceived worklife balance support. In: Asia Pacific Journal of Human Resources, 45(1). S. 113-123.

Maier, G. W./Woschée, R. W. (2002): Die affektive Bindung an das Unternehmen - Psychometrische Überprüfung einer deutschsprachigen Fassung des Organizational Commitment Questionnaire (OCQ) von Porter und Smith (1970). Zeitschrift für Arbeitsund Organisationspsychologie A\&O, 46 (3). S. 126-136.

Muster, V./Schrader, U. (2011): Green work-life balance: A new perspective for green HRM. In: German Journal of Research in Human Resource Management 25, 2/2011. S. 140-156.

Schrader, U./Harrach, C. (2013): Empowering Responsible Consumers to be Sustainable Intrapreneurs. In: Schrader, U./Fricke, V./ Doyle, D./Thoresen, V. (Hrsg.): Enabling Responsible Living, Berlin u. a. S. 181-192.

Spreitzer, G. (1995): Psychological empowerment in the workplace: Dimensions, measurement and validation. In: Academy of Management Journal, 38 (5). S. 1442-1465.

Valentine, S./Fleischman, G. (2008): Professional ethical standards, corporate social responsibility, and the perceived role of ethics and social responsibility. In: Journal of Business Ethics 82 (3). S. 657-666.

\section{AUTOREN + KONTAKT}

Christoph Harrach, Viola Muster und Laura Stanszus sind wissenschaftliche Mitarbeiter/innen am Fachgebiet Arbeitslehre/Ökonomie und Nachhaltiger Konsum der Technischen Universität Berlin; Ulf Schrader leitet dieses Fachgebiet.

Technische Universität Berlin, Fachgebiet Arbeitslehre/Ökonomie und Nachhaltiger Konsum, Marchstr. 23, 10587 Berlin. Tel.: +49 3031428769 , E-Mail: schrader@tu-berlin.de, Internet: http://www.aloenk.tu-berlin.de politische ökologie

Die Buchreihe für Querdenker und Vordenkerinnen

\section{Vom rechten Maß}

Suffizienz als Schlüssel zu mehr Lebensglück und Umweltschutz

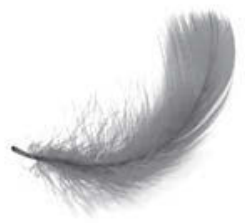

Noch ist die Kultur des „Weniger ist mehr" nicht im Mainstream angekommen, Appelle ans Maßhalten gelten als unpopulär. Doch immer mehr Menschen entdecken den Homo collaborans in sich und beweisen nebenbei, dass die Formel Suffizienz $=$ Verzicht falsch ist: In Reparaturcafés, Genossenschaften und Tauschbörsen leben sie vor, warum das Orientieren an den wahren Bedürfnissen glücklicher macht, wirtschaftlich sinnvoll ist und die natürlichen Ressourcen schont. Für die Neudefinition der Komfortzone braucht es aber auch politische Weichenstellungen für den Weg in einen suffizienten Alltag.

Mit Beiträgen von Niko Paech, Manfred Linz, Uwe Schneidewind, Angelika Zahrnt, Harald Heinrichs, Konrad Ott, Oliver Stengel,

Mathias Binswanger u.v.m.

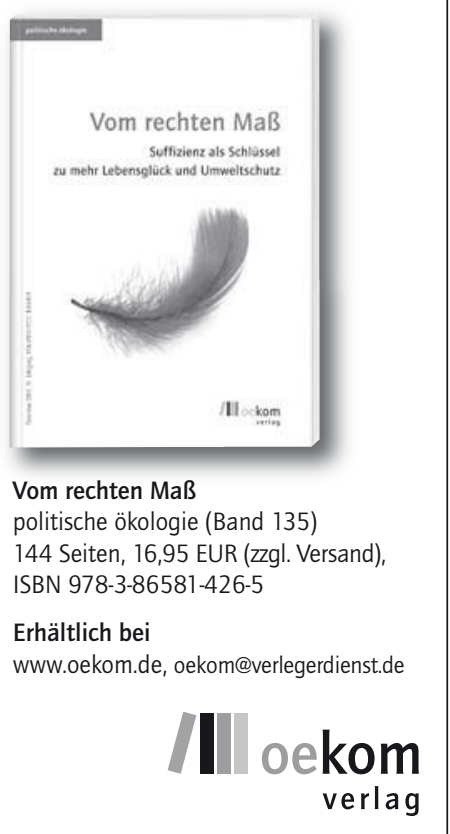

